\title{
Creating a Virtuous Cycle in Performance Testing at MongoDB
}

\author{
David Daly \\ MongoDB Inc \\ david.daly@mongodb.com
}

\begin{abstract}
It is important to detect changes in software performance during development in order to avoid performance decreasing release to release or dealing with costly delays at release time. Performance testing is part of the development process at MongoDB, and integrated into our continuous integration system. We describe a set of changes to that performance testing environment designed to improve testing effectiveness. These changes help improve coverage, provide faster and more accurate signaling for performance changes, and help us better understand the state of performance. In addition to each component performing better, we believe that we have created and exploited a virtuous cycle: performance test improvements drive impact, which drives more use, which drives further impact and investment in improvements. Overall, MongoDB is getting faster and we avoid shipping major performance regressions to our customers because of this infrastructure.
\end{abstract}

\section{CCS CONCEPTS}

- General and reference $\rightarrow$ Performance; • Information systems $\rightarrow$ Database performance evaluation; $\bullet$ Mathematics of computing $\rightarrow$ Time series analysis.

\section{KEYWORDS}

change point detection, performance, testing, continuous integration, variability

\section{ACM Reference Format:}

David Daly. 2021. Creating a Virtuous Cycle in Performance Testing at MongoDB. In Proceedings of the 2021 ACM/SPEC International Conference on Performance Engineering (ICPE '21), April 19-23, 2021, Virtual Event, France. ACM, New York, NY, USA, 9 pages. https://doi.org/10.1145/3427921.3450234

\section{INTRODUCTION}

Over the last several years we have focused on improving our performance testing infrastructure at MongoDB. The performance testing infrastructure is a key component in ensuring the overall quality of the software we develop, run, and support. It allows us to detect changes in performance as we develop the software, enabling prompt isolation and resolution of regressions and bugs. It keeps performance regressions from being included in the software we release to customers. It also allows us to recognize, confirm, and lock in performance improvements. As a business, performance testing impacts our top and bottom lines: the more performant the server,

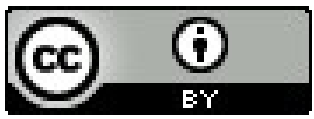

This work is licensed under a Creative Commons Attribution International 4.0 License.

ICPE '21, April 19-23, 2021, Virtual Event, France.

ACM ISBN 978-1-4503-8194-9/21/04.

https://doi.org/10.1145/3427921.3450234 the more our customers will use our services; the more effective our performance testing infrastructure, the more productive are our developers. Testing performance and detecting performance changes is a hard problem in practice, as performance tests and test platforms inherently contain some degree of noise. The use of change point detection [10] was a large improvement in our ability to detect performance changes in the presence of noise.

After putting our change point detection system into production, we explicitly focused on 4 challenges: how to deal with the large number of results and process all the changes; how to better deal with and isolate noise due to the testbed system itself; how to easily compare the results from arbitrary test runs; and how to capture and how to more flexibly handle more result types. The first two are familiar challenges, having been an explicit focus of the change point detection work, while the second two challenges become more serious problems once we achieved a basic ability to process our existing results.

The cumulative impact of these changes and our previous work has been to enable a virtuous cycle for performance at MongoDB. As the system is used more, we catch and address more performance changes, leading to us using the system more.

The rest of this paper is organized as follows. In Section 2 we review our previous work on which this paper builds. In Section 3 we discuss changes that have happened naturally as we have used the system more, leading to more load on the system. We then dive into four changes that we have tried in order to improve our infrastructure: Section 4 for improving our processing of results, Section 5 for handling more result types, Section 6 to address system noise, and Section 7 to improve the comparison of arbitrary test runs. Those sections are followed by a dive into the practical impact of all these changes in Section 8, before reviewing future work, related work, and conclusions in Sections 9, 10, and 12.

\section{REVIEW}

We built our performance testing infrastructure to be completely automated, and integrated with our continuous integration system Evergreen [3]. From past experience we had concluded that it was essential to automate the execution and analysis of our performance tests, and regularly run those tests as our developers worked on the next release. Previously we had done ad-hoc testing and manual testing at the end of the release cycle. In both cases we were continually challenged by test results that would not reproduce, as well as a huge diagnosis effort to identify which component and changes to that component caused the performance changes. The combination of those challenges led to a large effort late in each release cycle to try to identify and fix performance regressions, often resulting in release delays or performance regressions shipping to customers. Creating the infrastructure [16] to test performance in our CI system let us identify and address regressions earlier, and made it much easier to isolate performance changes. 


\begin{tabular}{lrrr}
\hline & 2018 & 2019 & 2020 \\
\cline { 2 - 4 } Number of Configurations & 8 & 17 & 24 \\
Number of Tasks & 86 & 181 & 356 \\
Number of Tests & 960 & 1849 & 3122 \\
Number of Results & 2393 & 3865 & 5787 \\
\hline
\end{tabular}

Table 1: The number of total possible test results we can create per source code revision has increased significantly over the past two years. This is due to increases in the number of tests and the number of configurations in which we run those tests.

Automation does not inherently make the tests reproducible, but it does make it clearer that there is noise in the results. Further work went into lowering the noise in the test results [14]. That work lowered, but did not eliminate the noise in the performance results. It was still challenging to detect changes in performance. Originally we tested for performance changes above some threshold (usually $10 \%$ ), but this had a number of problems, leading us to use change point detection [10]. Change point detection attempts to determine when there are statistical changes in a time-series, which is precisely the problem we want to solve. After the transition to change point detection, we had a system with completely automated, low noise tests that we could successfully triage and process.

\section{ORGANIC CHANGES}

There are a number of organic changes to our performance test environment that have occurred over the last couple of years. These changes were not planned, but they were still important changes. The performance testing system works, detecting that the performance has changed and correctly identifying when those performance changes occurred. The development engineers have seen that it works and so they use the performance test infrastructure more. One key aspect of that increase in use is that the development engineers have added more tests. We have also added new test configurations to further increase test coverage. Development engineers and performance engineers both add performance tests and configurations.

Table 1 shows the number of system under test configurations, tasks (collections of tests), tests, and number of raw results from running the performance tests for any version of the software. The data covers that past three years and is collected from tests run in September of each year. The table specifically filters out canary ${ }^{1}$ results and anything that we would not actively triage. In some cases, the line between configurations, tasks, tests, and results may be arbitrary, but it is how our system is organized and users interact with each of those levels.

You can see the huge increase in every dimension. We run our change point detection algorithm on the time-series for every result, and someone must triage all of that data. The total number of results went up 50\% year over year, and $142 \%$ over two years.

Additionally, the development organization has grown leading to more commits to our source repository. Overall the number of

\footnotetext{
${ }^{1}$ Canary tests are discussed in Section 6.
}

\begin{tabular}{lrrr}
\hline 12 months ending & 2018-09-01 & 2019-09-01 & 2020-09-01 \\
\hline Commits & 4394 & 4702 & 5538 \\
Commits per day & 12.0 & 12.9 & 15.2 \\
\hline
\end{tabular}

Table 2: The number of commits per day to our source repository has been increasing as the development organization has grown.

engineers working on our core server has gone up approximately $30 \%$ year over year for the past two years. Table 2 shows the number of commits and commits per day over the last 3 years. There has a been a steady increase in commits, going up $18 \%$ in the past year and $27 \%$ over the past two years. Each commit can potentially influence performance. If you combine the increased commit velocity with the increase in results per revision, you get a $76 \%$ increase in total results year over year, and an over $3 x$ increase in total possible results to generate and analyze over two years.

The net result of these changes (more commits + engineers using the system more) is many more possible results that may introduce performance changes and need to be isolated. During this time we have not increased the people dedicated to processing these results. All the problems we needed to address in the past are increased. Our processes to find and isolate changes need to scale or they will break down under the weight of new results.

\section{BETTER PROCESSING OF PERFORMANCE CHANGES}

In our previous paper [10] we described the role of "build baron": the "build baron" is a dedicated role to triage all performance changes, producing JIRA tickets and assigning them to the appropriate teams to address the changes. Originally the build baron role rotated through the members of the team that built the performance infrastructure. On the positive side, these people knew the system very well. However, that was balanced by the feeling that the work was a distraction from their primary work. Build baroning was a large transition from regular day to day work, and required both rebuilding mental state when becoming build baron and when returning to normal work. Everyone tried to dedicate the proper time to the work, but it is easy to want to do a little bit more of the development work you had been doing. Additionally, it's likely that the skills for a build baron differ from the skills of a software developer.

As such, we built a new team dedicated to build baroning. This new team originally covered correctness build failures, but has since expanded to the performance tests as well. The roles still rotate with the build baron team, but the team is always doing triage (not triage and development). The team members are better able to build up intuition and mental state about the system, and can more easily get help from each other. Possibly more importantly for members of this new team, triaging failures is their job, not an interruption from their job. While we added this new team, we did not allocate more people to doing the build baroning, rather we shifted who was doing the work.

The dedicated team is also able to better articulate the challenges of build baroning, and what changes would make them more productive. Over time the team developed a set of heuristics to 
deal with all the change points they had to process and shared knowledge. Part of this was adding filters to the existing boards and new ways of looking at the data. Where feasible we reviewed these heuristics and integrated them into the displays by default. Examples include better filtering of canary workloads (recall we do not want to triage changes in canaries, but rather rerun them) and sorting capabilities.

The impact of these changes show up in our overall statistics which are discussed in Section 8. The summary is that they allowed us to evaluate more tests and commits to find more changes, while also increasing the overall quality of the generated tickets without any additional human time.

\section{MAKING THE SYSTEM MORE DESCRIPTIVE}

Our performance testing environment was originally designed for tests that measured throughput, as throughput based tests are the easiest to create and analyze (just run an operation in a loop for a period of time, possibly with multiple threads). This assumption got built into the system. We knew it was a limitation in our system and have been striving to get around it. We developed conventions to add some latency results to our system, but it was inelegant. Worse, it largely assumed only one result per test. Ideally we could measure many performance results per test, such as throughput, median latency, tail latencies, and resource utilizations. Before change point detection, we could not add significantly more metrics since we could not keep up with the simpler tests we already had. Now that we had change point detection, we wanted to be able to track and process these additional metrics.

There were fundamentally two ways we could add these new metrics: 1 . Have tests measure the metrics of interest and then compute and report the relevant statistics to the results system. 2 . Have tests measure the metrics of interest and report all of those results to the result system. In the second case the test would report the metric for every operation - much more data - and let the results system calculate the statistics. After some review, we decided we preferred case 2, but that we also had to support case 1 .

We preferred the more data intensive case 2 because of what it enables. If we run a test that executes $10 \mathrm{k}$ operations, the system will report the latency for each of those 10k operations. First, having all the data allows us to change and recompute the statistics in the future. For example, if we decide we need the $99.99 \%$ latency in addition to the existing statistics, we can add it and recompute. If the test itself was computing the statistics we would have to rerun the test. Additionally, it allows us to view performance over test time, within a test and from the test's perspective (client side). This gives us a much more dynamic view of the performance of the system. We chose our preferred case, and it was paired with work on our open-source performance workload generation tool Genny [7]. We created a new service called Cedar [5] to store the results and calculate the statistics, and a tool called Poplar [6] to help report the results from the testbed to Cedar. Both are open source and part of our continuous integration system ecosystem [4]

While we chose the detailed case, we decided we also had to support the case in which tests computed their own statistics. The reason for this was simple: in addition to workloads written in
Genny, we also run third party industry standard benchmarks in our regression environment (e.g., YCSB $[8,9])$. Those tests already generate their own statistics, and it is not reasonable to adapt each such workload to report the metrics for every operation. The system we built handles both the case of getting all the raw results and the case of receiving the pre-aggregated data.

The new system was just that, a new system. We needed to integrate it into our production systems without breaking anything. The test result history is important both to the result display as well as the change point analysis, so we could not just turn off the old system and turn on the new. Instead we needed to make the old system and the new work together in the UI. We also needed to make it possible to handle the increase in information without completely overwhelming the build baron team ${ }^{2}$.

Figure 1 shows a snapshot of the existing build baron triage page and Figure 2 shows a snapshot of the new triage board. These pages are setup to enable the build barons to triage detected change points, create JIRA tickets, and assign those tickets to teams. We aggregate all change points for a given commit revision into one line by default to simplify processing. Each group of change points can be expanded to show all the impacted tests and configurations, as is done for one group in Figure 2.

For now we have placed all the new data on a new tab called "Change Points - Expanded Metrics". Adding a new tab is not optimal, but it does allow us to update and experiment with the new system with no fear of breaking our existing system and the processing of the legacy "Change Points" tab. Eventually we expect that the two tabs will merge together. The new tab has the additional column "Measurement". The argument in the field is a regular expression allowing tight control and filtering for the build baron. For now, the system is setup to display three such metrics (50th, 95th, and 99th percentile latencies). We expect to add more metrics to be triaged, as well as migrating the legacy metrics to this page in the future. The page also shows for each change the date the change was committed (Date) as well as the date on which the change point was calculated (Calculated On). The first is useful for understanding the development of the software, while the latter is useful for insight into the change point detection process. A change point that has been calculated recently is the result of more recent test executions. Both dates replace the somewhat ambiguous "create time" on the original page.

We also display trend graphs for each test, showing the evolution for a performance result over time, as the software is developed. The graphs are included on the page summarizing results for each task. As in the case of the triage page, we worried about overwhelming the users with additional results, so we added a pull down enabling the user to select which metric to display. Figure 3 shows a particularly interesting example of the value of these additional metrics and graphs. We detected a small change in average throughput, but further investigation showed a clearer change in the 90th percentile latency, while there was no change in the median latency. This information makes it easier to debug the issue, as it clearly is not the common path that is slower, but rather something making a small fraction of the operations significantly slower.

${ }^{2}$ The results discussed in this section are in addition to the increase in results discussed in Section 3 


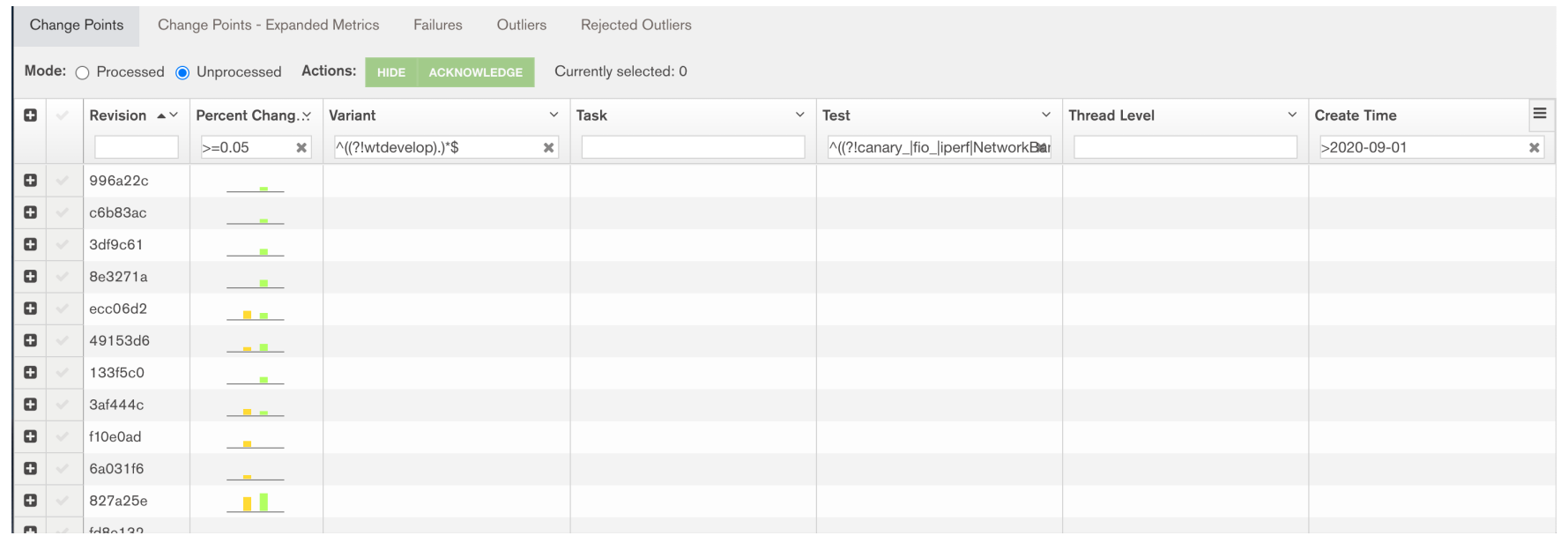

Figure 1: The existing build baron triage page is used by the build barons to triage change points on the existing data.

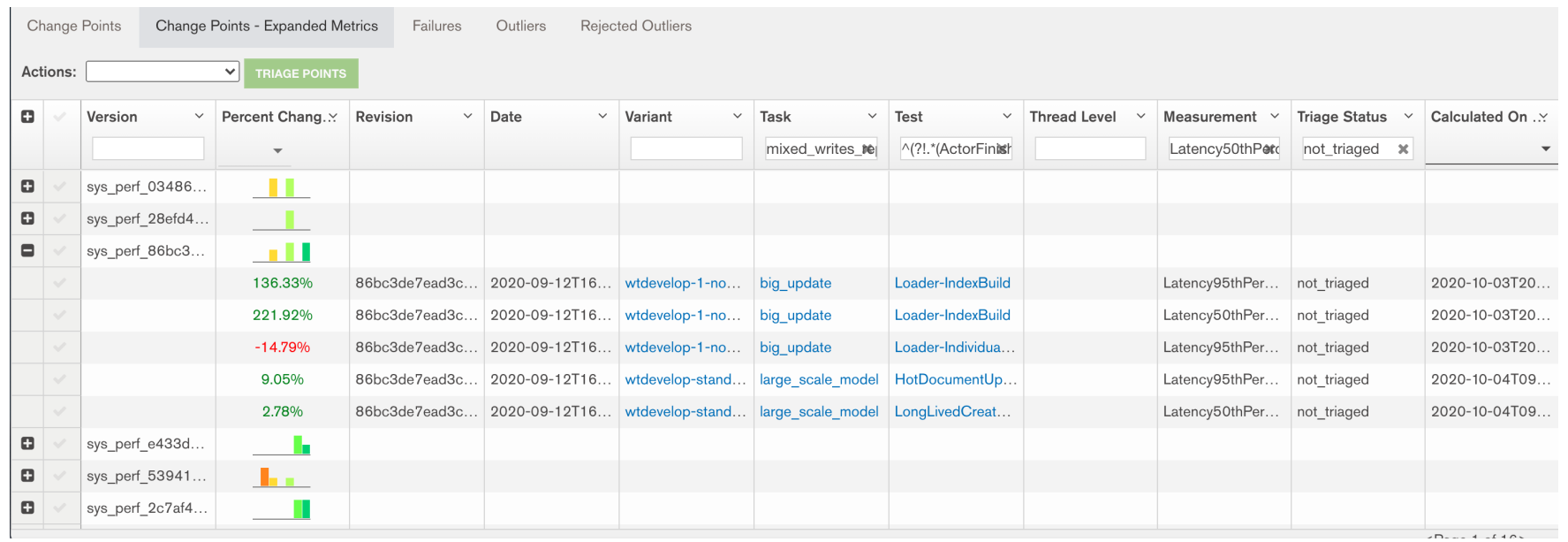

Figure 2: The new build baron triage page is used by the build barons to triage change points detected on the new, expanded metrics.

\section{LOWERING THE PERFORMANCE IMPACT OF SYSTEM ISSUES}

We run many of our performance tests in the Cloud and have done work to reduce the noise and increase the reproducibility of that system [14]. Sometimes there are performance problems on the testbed itself. We use "canary tests" to detect that. A "canary test" is a test that tests the testbed instead of software under test. In normal operation we expect the results for our canary tests not to change over time.

The canary tests are tests just like any other test, but treating them the same leads to some challenges. First, anyone looking at the result needs to know what is a canary test and what is not. We do not want server engineers spending any time diagnosing canary test failures. At the same time, we also do not want a server engineer diagnosing a performance change on a non-canary test when a canary test has also failed. Ideally, we would discard that result because it is suspect, and rerun those performance tests. If we were able to completely discard every (or even most) case of significant noise due to the system, it makes the job of the change point detection algorithm that much easier.

We set out to lower the impact of system noise by leveraging the data from our change point detection algorithm. We recognized that while changes in server performance manifested as changes in the distribution on our performance test results, system noise was different. The common problem was a bad run with results that did not match recent history. This is a problem of finding statistical outliers, not of finding change points. As NIST defines it, "An outlier is an observation that appears to deviate markedly from other observations in the sample." [1].

There are a number of existing outlier detection algorithms. We implemented the Generalized ESD Test (GESD) [2, 27] algorithm. The code is included in our open source signal processing repository (https://github.com/10gen/signal-processing). Specifically, we wanted to use the outlier detection to detect outliers on canary 


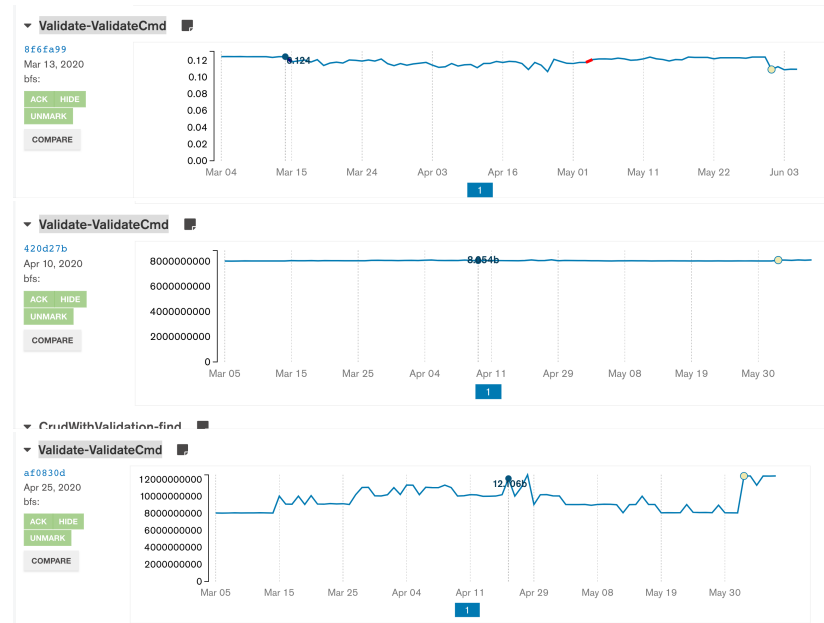

Figure 3: Three trend views of the same test, showing a performance regression. All three show performance over time. The top graph shows throughput, the middle shows median latency, and the bottom graph shows 90 th percentile latency. The regression is visible on the throughput and 90th percentile latency graphs, but not for the median latency.

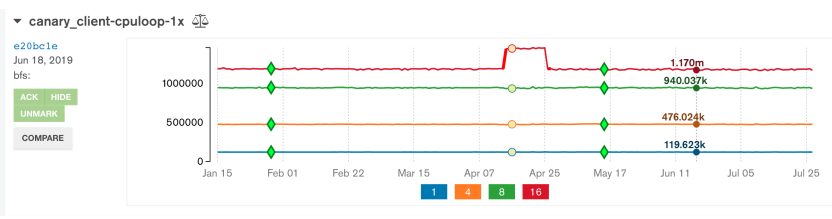

Figure 4: Performance for one of our canary workloads over time. It shows a real, if short lived, change in testbed performance, causing the outlier detection based system to rerun many tests.

tests. An outlier on a canary test would indicate something strange happened on the testbed. We want to not use the data from such a run, and ideally rerun those experiments. When an outlier is detected on a canary test, we would automatically suppress the test results for that task and reschedule the task.

While reasonable in theory, we ran into some challenges. Figure 4 shows an example of one such challenge: We had a short period of time in which the underlying system got faster. This may have been a temporary configuration change. Essentially every task that ran after that change was flagged as an outlier and re-run. In fact, they were all run 3 or more times. This cost a lot of money and (worse) slowed our ability to get results. Also, as it was a real change in the underlying testbed performance, the results did not noticeably change with any of the re-runs. In this case we spent a lot of money for no improvement. We added a system to "mute" such changes, but it required active intervention to avoid the worst cases. This change did not last long, but it was long enough to cause more outliers and re-runs when the performance returned to normal.

In other cases the system would rightly detect a transient change in testbed performance, but the underlying issue lasted for some period of time. The tests would immediately rerun, but still get the bad results. Only after waiting some period of time would the performance return to normal on a rerun.

At the end of the day we disabled the system. It was not solving our problem, but it was costing us money. We have kept the computation running, so we have built up a wealth of data when we come back to this area or decide to use outlier detection for other challenges.

\section{IMPROVED COMPARISON OF ARBITRARY RESULTS}

Our previous work on change point detection [10] only addressed identifying when performance changed. It did nothing for comparing two arbitrary builds to see if performance changed. There are two common cases in which we want to compare arbitrary test runs:

(1) Comparing performance from recent commits to the last stable release.

(2) Comparing performance from a "patch" build (proposed change). Does that patch change performance?

In the first case we want to determine the net performance change over a period of time. Very commonly this is how we check how our proposed release compares to the previous release. We would like to know what is faster, what is slower, and what is more or less stable now compared to then. There may be multiple changes in performance for a given test across a release cycle. Change point detection helps us understand each of those changes, but at the end of the day we need to let our customers know what to expect if they switch to the newer version. This check also provides a backstop to change point detection to make sure nothing significant has slipped through the triage process.

In the second case the engineer needs to know what impact their changes will have on performance. We have tools to compare the results from two arbitrary test executions, but it does not have any sense of the noise distribution for the test. It makes it hard to tell which differences are "real" and which are just artifacts of the noise of those particular runs. A common pattern to deal with this is to compare all the data, sort by percentage change, and inspect the tests with he largest changes. Invariably the largest reported changes are due to noise, usually from tests that report a low absolute result value (e.g., latency of something fast), leading to large percentage changes. An advanced user may learn which tests to ignore over time, while a less experienced user may either use brute force, or enlist an experienced user. Neither solution is a good use of time.

The change point detection system does not directly improve our ability to compare performance across releases, however, its results do enable smarter comparisons. All of the data from the change point detection algorithms is available in an internal database. That data includes the location of change points, as well as sample mean and variances for periods between change points. The sample mean averages out some of the noise, and the sample variance gives us a sense of how much noise there is. We can use that data a number of ways to improve the comparison. The simplest may be to compare means instead of points, and use the variance data to understand how big the change is relative to regular noise. 
After a few iterations we had the following system:

- Select two revisions to compare.

- Query the database for all the raw results for each revisions.

- For each result query the database for the most recent change point before the given revision. Save the sample mean and variance for the region after the change point.

- Compute a number of new metrics based on those results.

The new computed values were:

- Ratio of the sample means

- Percentage change of the sample means

- Change in means in terms of standard deviation

Note that there are better statistical tests we could use (see future work in Sec 9). Comparing means and standard deviations is not technically correct for determining the probability that a change is statistically significant. However, it is both easy to do and proved useful for a prototype.

We exported the data as a CSV file and operated on it in a spreadsheet for a first proof of concept. Our first instinct was to sort all the results by how many standard deviations a change represented, however, that did not work well. It turned out that some of our tests reported very low variances. The top results ended up being very small changes in absolute terms, but huge changes in terms standard deviation. With that in mind, we shifted to a more complex strategy: we filtered out all results that were less than a 2 standard deviation change, and then sorted by percentage change. We felt comfortable doing that since we did not need to catch every change for the current use, only the most significant (in a business sense, not a statistical one) changes. A change that was less than two standard deviations was unlikely to be the performance change that the engineering organization had to know about. Once we filtered on number of standard deviations and sorted on percentage change, the signal greatly improved. The most important changes rose to the top and were reviewed first.

We regularly need the ability to compare two commits as part of a monthly update on performance. Once a month we checkpoint the current status of performance for the development branch against the previous month, and against the last stable release. This gives us the big picture on the state of performance, in addition to the detailed results from change point detection. Figure 5 shows a spreadsheet we created using this process for a recent monthly checkpoint on the state of performance. The figure shows the two standard deviation filter applied ("Deviation" column), and then sorted on the "Percent Change" column. This view enabled us to quickly review all the real changes and avoid changes that were due to noisy tests. For example, the top test is $250 \%$ faster across the comparison. While we have shown performance improvements in the figure, we review both improvements and regressions to get a complete view of the state of performance.

In practical terms, this POC has lowered the cost of reviewing the monthly build from multiple hours, to somewhere between 30 and 60 minutes. Additionally, all of that time is now productive time looking at real issues. If there are more issues, it takes more time, and if there are fewer, it takes less time. We expect to transition this view from the CSV and proof of concept stage, into another page in our production system available to all engineers. We also expect to implement more rigorous statistical tests.

\begin{tabular}{lrr}
\hline Release Cycle & $\mathbf{4 . 2 . 0}$ & $\mathbf{4 . 4 . 0}$ \\
\hline Total Tickets & 273 & 393 \\
Resolved Tickets & 252 & 346 \\
Percent Resolved & $92.3 \%$ & $88.0 \%$ \\
Resolved by Release & 205 & 330 \\
Percent Resolved by Release & $75.1 \%$ & $84.0 \%$ \\
Release Duration & 412 & 352 \\
Tickets per Day & 0.66 & 1.12 \\
\hline
\end{tabular}

Table 3: $\overline{\text { Statistics on performance related JIRA tickets over }}$ the previous two release cycles.

\begin{tabular}{lrr}
\hline Release Cycle & $\mathbf{4 . 2 . 0}$ & $\mathbf{4 . 4 . 0}$ \\
\hline Code related & $28.57 \%$ & $43.06 \%$ \\
Test related & $8.73 \%$ & $7.80 \%$ \\
Configuration related & $0.00 \%$ & $0.58 \%$ \\
System related & $28.17 \%$ & $24.86 \%$ \\
Noise related & $7.94 \%$ & $6.94 \%$ \\
Duplicate ticket & $11.11 \%$ & $14.45 \%$ \\
Not labeled & $16.67 \%$ & $2.31 \%$ \\
\hline
\end{tabular}

Table 4: Breakdown of root causes for performance JIRA tickets.

\section{IMPACT}

The combination of the changes described above has had noticeable impact on our performance testing infrastructure and on our engineering organization. The basic way we track a performance change is a JIRA tickets. We compiled statistics from our JIRA tickets to quantify part of that impact. The statistics are aligned with our release cycle, which is nominally a year long.

We had considerably more performance related BF tickets in 4.4.0 than 4.2.0, over a shorter release cycle. Tickets per day went from 0.66 to 1.12 , a $70 \%$ increase. We had a large increase in tickets, but simultaneously increased the percentage of tickets resolved by the release. Those are both positive signs, especially since we spent the same amount of time triaging those changes, but it is only truly positive if the ticket quality has stayed the same or improved.

Table 4 shows quality information about our performance tickets. We label every ticket based on its cause. The best case is for the change to be code related: that indicates that the ticket captures a performance change based on changes in the code under test. These are tickets telling us something useful about our software. There are many other causes for tickets however. Performance changes can be created due to changes in the test (test related) or the testbed configuration (configuration related), the system itself can directly cause an error (system related), or noise in the system can create a false alert (noise related). Sometimes we create multiple tickets which we eventually determine are the same cause (duplicate ticket). Finally, some tickets are not labeled at all because they do not have a clear cause.

The fraction of code related tickets has gone up, even as the ticket volume has also gone up. We can conclude that we are generating more tickets, with the same amount of time dedicated to triage, and 


\begin{tabular}{|c|c|c|c|c|c|c|c|c|c|c|}
\hline 1 & task & $\nabla$ variant & test & threads & $\mid$ mean $x\lfloor\nabla \mid$ & $\operatorname{var} \mathrm{x}$ & meany $|\nabla|$ & J vary & Percent Change $-\downarrow \downarrow$ & Deviation \\
\hline 5 & out_of_cache_scanner & linux-1-node-15gbwt & ch HotSampler-Read.2 & 10 & o 29.5682396 & 1.14636286 & 68.52188063 & 80.3709177 & 246.9684793 & 19.65694035 \\
\hline 6 & out_of_cache_scanner & linux-1-node-15gbwt & ch HotSampler-ReadWithScan.2 & 10 & o 11.1117488 & 0.18634952 & 24.29358787 & 6.59059505 & 158.7986811 & 15.79439906 \\
\hline 7 & mongos_large_catalog_workloads & $s$ linux-shard-lite & mongos_large_catalog_1000000_colls_fresh_mongos & 1 & $1-3.7352941$ & 0.0768984 & $4-3886.1783$ & 4151.39418 & 99.90388258 & 14000.59346 \\
\hline 8 & mongos_large_catalog_workloads & $s$ linux-shard-lite & mongos_large_catalog_100000_colls_fresh_mongos & 1 & $1-3.4592105$ & 0.07430477 & $7-366.88627$ & 27.7684078 & 99.05714365 & 1333.241834 \\
\hline 9 & mongos_large_catalog_workloads & s linux-3-shard & mongos_large_catalog_1000000_colls_fresh_mongos & 1 & $1-62.326316$ & 9656.50316 & $6-4090.0813$ & 9321.96609 & $\mathbf{9 8 . 4 7 6 1 5 9 4 7}$ & 40.98765716 \\
\hline 10 & mongos_large_catalog_workloads & s linux-shard-lite & mongos_large_catalog_1000_colls_fresh_mongos & 1 & $1-2.9315789$ & 0.05032717 & -7.1125 & 0.08111111 & 58.7827213 & 18.63677299 \\
\hline 11 & change_streams_latency & linux-3-shard & 15_1c_max_latency & 4 & $4-1.6040268$ & 1.07863232 & $\begin{array}{ll}2 & -3.7619048\end{array}$ & 0.29047619 & 57.3613117 & 2.077734997 \\
\hline 12 & mongos_large_catalog_workloads & s linux-shard-lite & mongos_large_catalog_10000_colls_new_coll_same_db_mor & 1 & $1-2.3684211$ & 0.04870555 & $5-5.1744681$ & 0.04976873 & 54.22870499 & 12.71468867 \\
\hline 13 & mongos_large_catalog_workloads & $s$ linux-shard-lite & mongos_large_catalog_1000_colls_new_coll_same_db_mong & 1 & $1-2.3368421$ & 0.05860597 & $7-4.5617021$ & 0.07589269 & 48.77258445 & 9.190343563 \\
\hline 14 & mongos_large_catalog_workloads & $s$ linux-shard-lite & mongos_large_catalog_100000_colls_new_coll_same_db_mc & 1 & $1-2.4276316$ & 0.05982397 & $7-4.7190476$ & 0.05670151 & 48.55674757 & 9.368419698 \\
\hline 15 & mongos_large_catalog_workloads & s linux-shard-lite & mongos_large_catalog_1000000_colls_new_coll_same_db_m & 1 & -2.78 & 0.03326316 & -5 & 0.04444444 & 44.4 & 12.17226043 \\
\hline 16 & change_streams_multi_mongos & linux-3-shard & 15_1c_avg_latency & 60 & 0 -4.0416591 & 1.56695343 & $\begin{array}{ll}3 & -7.1534038 \\
-1\end{array}$ & 11.1851647 & 43.50019612 & 2.485855693 \\
\hline 17 & mongos_large_catalog_workloads & s linux-shard-lite & mongos_large_catalog_0_colls_new_coll_same_db_mongos & 1 & $1-2.7333333$ & 0.01066667 & $\begin{array}{l}7 \\
-4.6285714\end{array}$ & 0.05314286 & 40.94650206 & 18.35056395 \\
\hline 18 & agg-query-comparison & linux-wt-standalone & Aggregation.CountsFullCollection & & 42213.23188 & 10112.7905 & $5 \quad 1637.46489$ & 9027.30299 & 35.16209695 & 5.725471497 \\
\hline 19 & agg-query-comparison & linux-wt-standalone & Aggregation.DistinctWithoutIndexAndQuery & 4 & $4 \quad 444.319386$ & 506.287826 & $\begin{array}{ll}6 & 337.168544\end{array}$ & 181.60089 & 31.77960809 & 6.122527161 \\
\hline 20 & agg-query-comparison & linux-wt-standalone & Aggregation.DistinctWithoutIndexAndQuery & 8 & 8434.016626 & 5274.373206 & $\begin{array}{l}6 \quad 330.49747 \\
\end{array}$ & 177.844239 & 31.32222361 & 6.249566243 \\
\hline 21 & background_validate_cmd & linux-standalone & CrudWithoutValidation-find & 25 & 5216.122858 & 261.45351 & 1165.122818 & 302.170906 & 30.88612463 & 3.1540 \\
\hline 22 & background_validate_cmd & linux-standalone & CrudWithoutValidation-Crud & 25 & $5 \quad 216.069714$ & 261.347262 & 2165.083164 & 301.979203 & 30.88537264 & 3.153890795 \\
\hline
\end{tabular}

Figure 5: Spreadsheet view of performance comparing performance of two commits, taking advantage of the statistics generated by the change point detection algorithm.

\begin{tabular}{lrr}
\hline Release Cycle & $\mathbf{4 . 2 . 0}$ & $\mathbf{4 . 4 . 0}$ \\
\hline Performance improvements & 21 & 40 \\
Percentage of tickets that are improvements & $7.69 \%$ & $10.18 \%$ \\
Days per performance improvement & 19.62 & 8.80 \\
Performance regressions & 15 & 13 \\
Percentage of tickets that are regressions & $5.49 \%$ & $3.31 \%$ \\
Days per performance regression & 27.47 & 27.08 \\
\hline
\end{tabular}

Table 5: Breakdown on the number and rate of performance JIRA tickets closed as improvements and regressions over the past two release cycles.

the tickets are of higher quality than last year. In other words, we are doing our job better than last year. While we are happy with that improvement, we also recognize that less than half our tickets are about changes in the software under test. We would like to continue to drive that percentage higher.

Interestingly, the category with the largest drop are tickets that are not labeled. This is due to us doing a better job of diagnosing tickets and making them actionable. It is not the case that we were just missing code related tickets with the labels in the past. The number of duplicates is the only non-code related category to go up noticeably. We attribute this to the increase load of change points and tickets on the build barons.

The last measure of goodness is how many tickets were fixed (or not), and how many things improved. Table 5 shows those statistics. Before discussing the numbers we note that we count any net improvement as an improvement and any net regression closed without fixing as a regression, regardless of its practical significance. We had comparable number of accepted regressions year over year, while nearly doubling the number of improvements. So, even with the large increase in tickets, we still only get a regression that is not fixed about once a month, and we went from getting an improvement every 20 days to one every 9 days.

Clearly our system is working better. We have more tickets and they are higher quality. In addition to each component performing better, we believe that we have enabled a virtuous cycle. Performance issues get diagnosed faster, making them easier to fix, so more issues get fixed. Development engineers get used to receiving performance tickets and know they are high quality and operational. Since the system provides useful information, engineers are more likely to look to fix their regressions and to add more performance tests. As we add more performance tests, we are more likely to catch performance changes. One last improvement is that with increased trust, engineers are more likely to test their key changes before merging, so we can avoid some performance regressions ever being committed to the development mainline.

\section{FUTURE WORK AND CONTINUING CHALLENGES}

Our current performance testing system enables us to detect performance changes during the development cycle, and to enable our developers to understand the impact of their code changes. While we have made great progress, there is still much that we would like to improve in the system. We expect that everything (commits, tests, results, changes) will continue to increase, putting more load on our system. Additionally, we are increasing our release frequency to quarterly [21], which will further increase the load on the system. In the near term we are working to improve the ability to compare arbitrary versions, building on the work described in Section 7. This will involve both using better statistical tests, such as Welch's t-test [28] (assuming normality) or Mann-Whitney U-test [19] in place of the simple variance based calculation, as well as building the view into our production system. This will help us to compare performance between releases, as well as help developers determine if their proposed changes impact performance.

There is still much we can do on the change point detection itself. In order to simplify the implementation, all tests and configurations are treated as separate and independent time series by the change point detection algorithm. We think there is a large opportunity to consider correlations between tests and configurations. It is very infrequent that one test and configuration changes separately from all others. We should be able to exploit correlated changes to better diagnose and describe real performance changes, and exclude noise.

There is still too much noise in the system, including some cases of particularly nasty noise. Two examples include tests that show bimodal behavior and unrelated system noise. Some tests will return one of two different results, and may stay with one of those 
results for a period of time before reverting to the other (e.g., 5 tests runs at 20 followed by 4 tests runs at 10). The change point detection algorithm has a very hard time with bimodal behavior as it looks like a statistical change. Today, a human has to filter these changes out. There are also cases of system noise that are real performance changes due to compiler changes. Sometimes these are due to code layout issues letting a critical code segment fit within or not fit within a performance-critical hardware cache. These issues manifest as deterministic changes in performance, but there is not much we can do about them except filter them out by hand.

Ultimately, the goal of all of this work can be described as a multidimensional optimization problem. We want to simultaneously:

- Maximize the useful signal on performance versus noise and distractions.

- Maximize the test and configuration coverage.

- Minimize the cost of performance testing.

- Minimize the time from creation of a performance change to its detection, diagnosis, and fix. (the limit of this is catching a regression before commit).

We have work to do on all of these points. Often, in the past, we have found ourselves with bad options, which explicitly trade off one point for another. We hope to develop techniques that improve one or more items above at the same time, without hurting the others.

\section{RELATED WORK}

Related work has looked at testing performance in continuous integration systems. Rehman et al. [26] describe the system developed for testing SAP HANA and stressed the need for complete automation. The system compared results to a user specified limit in order to determine a pass fail criterion. The authors also discuss challenges in reproducibility, isolation, and getting developers to accept responsibility for issues.

Continuous integration tests need to be fast, but standard benchmarks require extended periods of time to run. Laaber and Leitner [17] looked at using microbenchmarks for performance testing in continuous integration to deal with this problem. They found some, but not all microbenchmarks are suitable for this purpose.

Once performance tests are included in a CI system, the next challenge is to efficiently isolate the changes. Muhlbauer et al. [22] describe sampling performance histories to build a Gaussian Process model of those histories. The system decides which versions should be tested in order to efficiently build up an accurate model of performance over time and to isolate abrupt performance changes. The paper addresses a problem similar to our previous work on detecting change points in test histories [10], although our previous work assumes performance test results have a constant mean value between change points.

Test result noise is an ongoing challenge. Several papers investigate both sources of noise $[12,20]$ and quantifying the impact of that noise [18]. Duplyakin et al. [12] use change point detection to identify when the performance of the nodes in a datacenter change. Their objective is to identify and isolate those performance changes in order to keep them from impacting experiments run in the datacenter. The paper by Maricq et al. [20] includes a number of practical suggestions to reduce performance variability. The suggestions should be useful for anyone running performance benchmarks, and we perform many of these suggestions in our system. They also show the lack of statistical normality in their results, validating our design choice to not assume normality. Finally, Laaber et al. [18] compare the variability of different microbenchmark tests across different clouds and instance types on those clouds, demonstrating that different tests and different instance types have wildly different performance variability. Running benchmark test and control experiments on the same hardware can help control the impact of that noise.

The related area of energy consumption testing shows similar issues with test noise. Ournani et al. [24] describe the impact of CPU features (C-states, TurboBoost, core pinning) on energy variability. We have observed similar impacts on performance variability from those factor in our test environment [14]. Other work looks at extending the state of the art for change point detection in the presence of outliers [25]. Our system is sensitive to outliers in the results as well. Our efforts on outlier detection would have helped reduce the impact of outliers in our use case, if it had been successful.

Finally, there is ongoing work related to our ultimate goal of more efficiently detecting changes while simultaneously increasing our overall performance test coverage. Grano et al. [13] investigated testing with fewer resources. While this work is focused on correctness testing, the principles can be extended to performance testing. Multiple papers $[11,15]$ try to identify which software changes are most likely to have performance impact in order to prioritize the testing of those changes. Huang et al. [15] use code analysis of software changes to decide which changes are most likely to impact which tests, while de Oliveria et al. [11] use many indicators (including static and dynamic data) to build a predictor of the likelihood of a performance change in the tests based on a given software change. Other work has focused on efficiently finding performance changes across both versions and configurations [23] and is specifically focused on minimizing test effort while enabling the testing of potentially huge space of configuration options and software changes. We hope to build on these efforts to improve the efficiency of our performance testing.

\section{ACKNOWLEDGMENTS}

The work described in this paper was done by a large collection of people within MongoDB. Key teams include the Decision Automation Group (including David Bradford, Alexander Costas, and Jim O'Leary) who are collectively responsible for all of our analysis code, the Server Tooling and Methods team who own the testing infrastructure, the Evergreen team which built Cedar and Poplar for the expanded metrics support, and of course our dedicated build baron team whom make the whole system work.

We would also like to thank Eoin Brazil for his feedback on drafts of this paper.

\section{CONCLUSION}

In this paper we have reviewed a number of recent changes to our performance testing infrastructure at MongoDB. This builds on previous work we have done to automate our performance testing environment, reduce the noise in the environment (both actual 
noise and its impact), and better makes use of the results from our performance testing. This infrastructure is critical to our software development processes in order to ensure the overall quality of the software we develop.

We first reviewed the general increase in load on the infrastructure. Each year we run more tests in more configurations while our developers commit more changes to our source repository. Overall we had a more than $3 x$ increase over two years in the total possible number of test results to generate and analyze.

Paired with the general increase in load, we focused on improving the scalability of our ability to process those results and isolate performance changes. We also added the ability to report more and more descriptive results from tests, enabling saving information about every operation within a performance test. This required new systems to store and process the results, as well as new displays for triaging the results.

Attempting to better control system noise, we built a system to detect when the performance of our testbeds changed, and therefore we should not trust the results of our performance tests. While promising in theory, in practice this did not work as well as we had hoped, and ultimately we disabled it.

Finally, we enabled better comparison of results between arbitrary commits. This was a large open challenge for us. Building upon the change point detection system we use to process our results, we were able to give a much clearer view of the significant changes between arbitrary commits, making it much easier to regularly check the current state of the development software against the last stable release. We continue to both refine this comparison of results and lift it into our production environment.

The cumulative impact of these changes and our previous work has been to enable a virtuous cycle for performance at MongoDB. As the system is used more, we catch and address more performance changes, leading us to use the system more. This virtuous cycle directly increases the productivity of our development engineers and leads to a more performant product.

\section{REFERENCES}

[1] [n.d.]. 1.3.5.17. Detection of Outliers. https://www.itl.nist.gov/div898/handbook/ eda/section $3 /$ eda35h.htm

[2] [n.d.]. 1.3.5.17.3. Generalized Extreme Studentized Deviate Test for Outliers. https://www.itl.nist.gov/div898/handbook/eda/section3/eda35h3.htm

[3] [n.d.]. Evergreen Continuous Integration: Why We Reinvented The Wheel. https://engineering.mongodb.com/post/ evergreen-continuous-integration-why-we-reinvented-the-wheel

[4] [n.d.]. Evergreen Ecosystem. https://github.com/evergreen-ci/evergreen

[5] [n.d.]. Package cedar. https://godoc.org/github.com/evergreen-ci/cedar

[6] [n.d.]. Package poplar. https://godoc.org/github.com/evergreen-ci/poplar

[7] 2020. Genny workload generator. https://github.com/mongodb/genny originaldate: 2018-02-12T19:23:44Z.

[8] 2020. YCSB. https://github.com/mongodb-labs/YCSB original-date: 2015-0317T18:10:30Z.

[9] Brian F. Cooper, Adam Silberstein, Erwin Tam, Raghu Ramakrishnan, and Russell Sears. 2010. Benchmarking cloud serving systems with YCSB. In Proceedings of the 1st ACM symposium on Cloud computing - SoCC '10. ACM Press, Indianapolis, Indiana, USA, 143. https://doi.org/10.1145/1807128.1807152

[10] David Daly, William Brown, Henrik Ingo, Jim O’Leary, and David Bradford. 2020. The Use of Change Point Detection to Identify Software Performance Regressions in a Continuous Integration System. In Proceedings of the ACM/SPEC International Conference on Performance Engineering (ICPE '20). Association for Computing Machinery, Edmonton AB, Canada, 67-75. https://doi.org/10.1145/ 3358960.3375791

[11] Augusto Born De Oliveira, Sebastian Fischmeister, Amer Diwan, Matthias Hauswirth, and Peter F. Sweeney. 2017. Perphecy: Performance Regression
Test Selection Made Simple but Effective. In 2017 IEEE International Conference on Software Testing, Verification and Validation (ICST). 103-113. https: //doi.org/10.1109/ICST.2017.17

[12] Dmitry Duplyakin, Alexandru Uta, Aleksander Maricq, and Robert Ricci. 2020. In Datacenter Performance, The Only Constant Is Change. In 2020 20th IEEE/ACM International Symposium on Cluster, Cloud and Internet Computing (CCGRID). 370-379. https://doi.org/10.1109/CCGrid49817.2020.00-56

[13] Giovanni Grano, Christoph Laaber, Annibale Panichella, and Sebastiano Panichella. 2019. Testing with Fewer Resources: An Adaptive Approach to Performance-Aware Test Case Generation. IEEE Transactions on Software Engineering (2019), 1-1. https://doi.org/10.1109/TSE.2019.2946773 arXiv: 1907.08578.

[14] Henrik Ingo and David Daly. 2019. Reducing variability in performance tests on EC2: Setup and Key Results. https://engineering.mongodb.com/post/ reducing-variability-in-performance-tests-on-ec2-setup-and-key-results

[15] Peng Huang, Xiao Ma, Dongcai Shen, and Yuanyuan Zhou. 2014. Performance regression testing target prioritization via performance risk analysis. In Proceedings of the 36th International Conference on Software Engineering (ICSE 2014). Association for Computing Machinery, Hyderabad, India, 60-71. https://doi.org/10.1145/2568225.2568232

[16] Henrik Ingo and David Daly. 2020. Automated system performance testing at MongoDB. In Proceedings of the workshop on Testing Database Systems (DBTest '20). Association for Computing Machinery, New York, NY, USA, 1-6. https: //doi.org/10.1145/3395032.3395323

[17] Christoph Laaber and Philipp Leitner. 2018. An evaluation of open-source software microbenchmark suites for continuous performance assessment. In Proceedings of the 15th International Conference on Mining Software Repositories (MSR '18). Association for Computing Machinery, Gothenburg, Sweden, 119-130. https://doi.org/10.1145/3196398.3196407

[18] Christoph Laaber, Joel Scheuner, and Philipp Leitner. 2019. Software microbenchmarking in the cloud. How bad is it really? Empirical Software Engineering 24, 4 (Aug. 2019), 2469-2508. https://doi.org/10.1007/s10664-019-09681-1

[19] H. B. Mann and D. R. Whitney. 1947. On a Test of Whether one of Two Random Variables is Stochastically Larger than the Other. Annals of Mathematical Statistics 18, 1 (March 1947), 50-60. https://doi.org/10.1214/aoms/1177730491 Publisher: Institute of Mathematical Statistics.

[20] Aleksander Maricq, Dmitry Duplyakin, Ivo Jimenez, Carlos Maltzahn, Ryan Stutsman, and Robert Ricci. 2018. Taming Performance Variability. In 13th $\$ \backslash\{\$$ USENIX\$1\}\$ Symposium on Operating Systems Design and Implementation (\$ $\mid\{\$ O S D I \$ \mid \$ 18) .409-425$. https://www.usenix.org/conference/osdi18/ presentation/maricq

[21] Mat Keep and Dan Pasette. 2020. Accelerating Delivery with a New Quarterly Release Cycle, Starting with MongoDB 5.0 | MongoDB Blog. https://www. mongodb.com/blog/post/new-quarterly-releases-starting-with-mongodb-5-0

[22] Stefan Muhlbauer, Sven Apel, and Norbert Siegmund. 2019. Accurate Modeling of Performance Histories for Evolving Software Systems. In 2019 34th IEEE/ACM International Conference on Automated Software Engineering (ASE). IEEE, San Diego, CA, USA, 640-652. https://doi.org/10.1109/ASE.2019.00065

[23] Stefan Mühlbauer, Sven Apel, and Norbert Siegmund. 2020. Identifying Software Performance Changes Across Variants and Versions. In 2020 35th IEEE/ACM International Conference on Automated Software Engineering (ASE). 12. https: //doi.org/10.1145/3324884.3416573

[24] Zakaria Ournani, Mohammed Chakib Belgaid, Romain Rouvoy, Pierre Rust, Joel Penhoat, and Lionel Seinturier. 2020. Taming Energy Consumption Variations In Systems Benchmarking. In Proceedings of the ACM/SPEC International Conference on Performance Engineering (ICPE '20). Association for Computing Machinery, New York, NY, USA, 36-47. https://doi.org/10.1145/3358960.3379142

[25] Paul Fearnhead and Guillem Rigaill. 2019. Changepoint Detection in the Presence of Outliers. 7. Amer. Statist. Assoc. 114, 525 (2019), 169-183. https://doi.org/10. 1080/01621459.2017.1385466

[26] Kim-Thomas Rehmann, Changyun Seo, Dongwon Hwang, Binh Than Truong, Alexander Boehm, and Dong Hun Lee. 2016. Performance Monitoring in SAP HANA's Continuous Integration Process. ACM SIGMETRICS Performance Evaluation Review 43, 4 (Feb. 2016), 43-52. https://doi.org/10.1145/2897356.2897362

[27] Bernard Rosner. 1983. Percentage Points for a Generalized ESD Many-Outlier Procedure. Technometrics 25, 2 (1983), 165-172. https://doi.org/10.2307/1268549 Publisher: [Taylor \& Francis, Ltd., American Statistical Association, American Society for Quality].

[28] B. L. Welch. 1947. The Generalization of 'Student's' Problem When Several Different Population Variances are Involved. Biometrika 34, 1-2 (Jan. 1947), 28-35. https://doi.org/10.1093/biomet/34.1-2.28 Publisher: Oxford Academic. 